\title{
Transatlantica
}

Revue d'études américaines. American Studies Journal

1 | 2018

Slavery on Screen / American Women Writers Abroad: 1849-1976

\section{Là où réside la mémoire: Sally Mann au Jeu de Paume}

Sally Mann : Mille et un passages, 18/06/2019-22/09/2019, Jeu de Paume (Paris)

\section{Faustine Rondin}

\section{(2) OpenEdition}

\section{Journals}

Édition électronique

URL : https://journals.openedition.org/transatlantica/12896

DOI : $10.4000 /$ transatlantica. 12896

ISSN : 1765-2766

Éditeur

Association française d'Etudes Américaines (AFEA)

Référence électronique

Faustine Rondin, «Là où réside la mémoire : Sally Mann au Jeu de Paume », Transatlantica [En ligne], 1 | 2018, mis en ligne le 23 septembre 2010, consulté le 03 février 2023. URL : http://

journals.openedition.org/transatlantica/12896; DOI : https://doi.org/10.4000/transatlantica.12896

Ce document a été généré automatiquement le 3 février 2023.

\section{c) $(i) \Theta$}

Creative Commons - Attribution - Pas d'Utilisation Commerciale - Pas de Modification 4.0 International - CC BY-NC-ND 4.0

https://creativecommons.org/licenses/by-nc-nd/4.0/ 


\section{Là où réside la mémoire : Sally Mann au Jeu de Paume}

Sally Mann : Mille et un passages, 18/06/2019 - 22/09/2019, Jeu de Paume (Paris)

\section{Faustine Rondin}

1 Les salles qui accueillent la rétrospective dédiée à l'œuvre de la photographe américaine Sally Mann sont baignées d'une douce lumière qui donne aux visiteurs le sentiment de marcher à l'ombre des arbres un jour d'été et qui confère à l'exposition une atmosphère semblable à celle d'un songe. L'impression de promenade au crépuscule est renforcée par le titre même de l'exposition qui insiste sur la notion de passage, notamment celui du temps, au cœur de cette rétrospective. Mais plus que son passage, ce sont ses traces qui semblent intéresser Mann, qui traque et capture les cicatrices, les indices, les empreintes, les souvenirs qu'il laisse sur les corps et sur les paysages. Les photographies de Mann oscillent entre tendresse et cruauté, beauté des paysages et violence du passé. Mann produit ainsi un corpus qui regorge de tensions intrinsèques à l'expérience de la vie, reflète à la fois les dissonances propres à sa terre natale (le Sud des États-Unis) mais aussi celles des "passages» de toute existence humaine. À travers le prisme du particulier, Mann interroge l'universel. La lumière tamisée qui nous accompagne le long du voyage photographique - et littéraire - que Mann nous propose est à la fois ode et requiem. Si, comme l'indique Mann, son Sud " est hanté par la mort », elle la dérange pour mieux interroger le paysage dans un acte qui vise à «faire rendre ses fantômes à la terre » (catalogue de l'exposition, 2019, 116 \& 42).

2 La rétrospective itinérante présentée au Jeu de Paume est la plus importante jamais consacrée à la production artistique de Mann. Elle s'articule autour d'un lieu, le Sud des États-Unis, terre mystérieuse, magnétique et hostile. Les deux commissaires de l'exposition, Sarah Greenough et Sarah Kennel, ont choisi de scinder l'œuvre de Mann en cinq séries: «La famille», "La terre », "L'ultime et pleine mesure», " Demeure avec moi », «Ce qui reste ». Le fleuve, lieu de passage par excellence, fait office de fil conducteur. Les cours d'eau élaborent une géographie sinueuse qui devient métaphore 
méandrique de la vie et de la mort. L'œuvre de Mann se découvre, se lit, comme un poème liquide.

3 Les clichés consacrés à sa famille - publiés pour la plupart dans l'ouvrage Immediate Family en 1992 - constituent, sans doute, le pan le plus connu de l'œuvre de Mann notamment parce que leur publication coïncide avec les "guerres culturelles » (des débats de société sur divers sujets tels que le rôle de l'art, l'homosexualité ou encore l'avortement) qui ont ébranlé les États-Unis dans les années 1990. Mann brosse un portrait de la vie domestique dépourvue des stéréotypes généralement liés à l'enfance. Elle photographie ses trois enfants, Jessie, Emmett et Virginia, dans un état prélapsaire: nus, insouciants, libres. L'iconographie religieuse tient une place importante dans cette série comme le démontrent des clichés tels que The Ditch (1987), Trumpet Flowers (1991) ou Emmett Floating at Camp (1991). Mais la photographe dépeint également l'enfance dans toute sa complexité : monde énigmatique fait de joies et de peurs, ponctué d'instants de grâce voués à disparaître (Jessie at Nine, 1991). Elle ne nous donne pas à voir les traditionnels rites de passage que sont les anniversaires ou les communions, mais d'autres pratiques, d'autres "cérémonies" pourrait-on dire pour emprunter un terme cher à Diane Arbus, non moins constitutives du monde de l'enfance: les blessures, les jeux de rôles (qui s'expriment à travers les poses empruntées au monde des adultes et les déguisements, comme c'est le cas, par exemple, dans Gorjus, 1989), les siestes, les bouderies.

4 Peu à peu, les corps abdiquent au profit des paysages. Mann sillonne le Sud pour capturer les champs de bataille de la guerre de Sécession, les marais, ou l'itinéraire d'Emmett Till, que Drew Gilpin Faust, qualifie de «pèlerinage visuel» ou encore de "rédemption photographique » (ibid. 132). Le vocabulaire sacré que la critique utilise montre bien à quel point il est important pour Mann de mettre le Sud face à son passé douloureux : sa photographie ne rachète pas les actes commis mais fait persister leur souvenir. Les clichés deviennent des injonctions, presque des prières : le passé ne doit pas se répéter mais ne doit pas non plus s'oublier.

5 La nature est le témoin silencieux de l'histoire, à l'image de cet arbre dont le tronc est entaillé horizontalement. Bien que l'arbre soit un sujet pictural classique, celui que Mann choisit d'immortaliser a la particularité d'être doté d'un certain anthropomorphisme. La cicatrice, seul élément net de la composition, donne l'impression que l'arbre est doté d'une bouche. Ses contours sombres semblent évoquer le sang coagulé. Mann opte pour le vignettage: les bords de la photographie sont assombris et permettent de se concentrer encore davantage sur cet arbre. La périphérie fuligineuse et le flou qui s'étale autour du tronc comme une brume ont pour effet de créer un vortex et confèrent au sujet une qualité onirique. Si la nature impassible est la gardienne mutique de la mémoire, la photographie, elle, permet de construire un passage, d'ouvrir une brèche, entre l'ici et l'ailleurs, le présent et le passé. 
Sally Mann, Deep South, Untitled (Scarred Tree), 1998,

Gelatin silver print

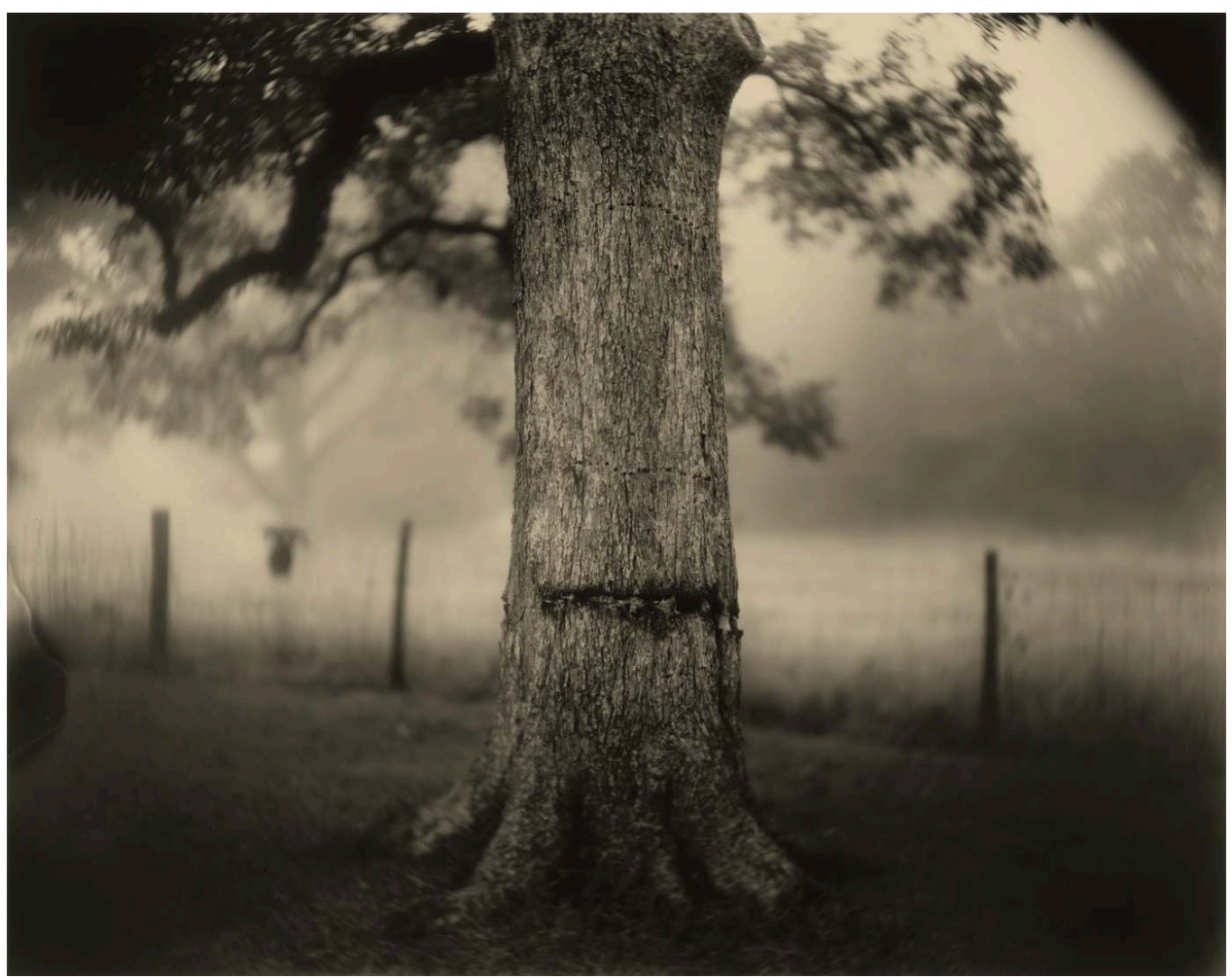

National Gallery of Art, Washington, Alfred H. Moses and Fern M. Schad Fund @ Sally Mann

Pour réaliser la majorité de ses clichés, Sally Mann, équipée de son encombrante chambre (8x10), a fait le choix d'utiliser un procédé inventé au XIXe siècle : le collodion humide. Le film qui clôt l'exposition, intitulé "Sally Mann: Collodion and the Angel of Uncertainty" (en référence à Proust et à "son ange de la certitude»), présente la photographe dans son atelier aux allures de laboratoire, doté de « collections de chimie ésotériques et explosives » (Hilton Als, 249). Mann y manipule la plaque de verre, qu'elle enduit de liquide sirupeux (le collodion) et qu'elle incline avec dextérité pour répandre le produit, un rituel qu'elle qualifie elle-même d'«expérience presque religieuse " (ibid. 251). Mais tandis que ses prédécesseurs utilisaient ce procédé pour obtenir une image dépourvue de défauts, Mann les accueille, prenant le parti, très tôt dans sa carrière, de travailler à partir de matériel défectueux, d'objectifs abîmés et de papier périmé. Le résultat obtenu, fruit de son travail mais aussi, dans une certaine mesure, du hasard, contient souvent stries, éraflures, éclaboussures. Ce n'est pas seulement l'aspect esthétique qui intéresse Mann mais la production de sens apportée par ces imperfections : les stries qui rayent certains clichés rappellent les échanges de tirs nourris entre les états confédérés et l'Union, les grains de poussière déposés sur une autre plaque forment des étoiles, c'est-à-dire des astres morts, tandis que les bordures noires inégales qui grignotent certains tirages font figure de présages funestes, qui simulent le passé. La photographe repousse sans cesse les limites de sa technique en créant des paysages inquiétants, d'un noir profond, qui tendent vers l'abstraction, comme nous pouvons le constater avec, par exemple, la série "Blackwater" où la nature photographiée devient une géométrie complexe. Les 
photographies, dont la lisibilité est difficile, deviennent des énigmes que le visiteur doit prendre le temps de déchiffrer.

7 L'exposition se poursuit par les photographies émouvantes de Virginia «Gee-Gee » Carter (morte à l'âge de 100 ans en 1994), femme afro-américaine qui, durant cinquante ans, a travaillé au service de la famille Munger (les parents de Sally Mann). Deux larges panneaux regroupent pêle-mêle soixante-dix photographies qui retracent la vie de Gee Gee. Ces clichés, de type amateur, dont certains ont probablement été glanés auprès de la famille de Virginia (comme semble le démontrer le film diffusé au centre de la pièce où l'on peut voir Mann entourée de deux des petites filles de Gee Gee), sont un hommage à celle qui a joué un rôle essentiel dans la vie de Sally Mann. En effet, cette femme adorée lui a permis d'amorcer une réflexion sur la nature des rapports qui unissent les blancs et les noirs dans le Sud. Cette série dédiée à Gee Gee vient alors dialoguer avec les monumentaux portraits d'hommes afro-américains (Janssen, Ronald, Stephen, Anton) réalisés entre 2006 et 2015, la série «Blackwater» (2008-2012) et les églises afro-américaines (2008-2016). Mann tente de réconcilier, de créer une voie (voire, une voix), un " passage » entre blancs et noirs à travers les corps (ceux de Gee Gee et de son homonyme, Virginia, la fille de Mann, et les portraits d'hommes afroaméricains) mais également à travers les paysages qui portent encore la marque, les stigmates, de l'esclavage et de la ségrégation. Cependant, ce sont les lieux qui incarnent une forme de résistance que Mann choisit de capturer : les marais où s'enfonçaient, au péril de leur vie, les esclaves en quête de liberté et les églises (Beulah Baptist, First Baptist Church of Goshen ou encore Payne's Chapel United Methodist qui disparaît sous l'épaisse végétation) où ils s'échappaient grâce à leur foi. Ces églises, exemples d'architecture vernaculaire (photographiés avec une pellicule orthochromatique) sont, contrairement à la plupart des clichés de Mann, tirés sur un papier périmé de petit format (d'où la diversité des teintes obtenues). La disposition de ces photographies, présentées en groupes, convoquent à l'esprit les châteaux d'eau et silos à grain immortalisés par le couple Becher. L'église devient un motif qui se répète mais, plus que le bâtiment, c'est son interaction avec la lumière qui interpelle. À la différence des Becher qui composaient un catalogue scientifique, Mann dresse un inventaire où point la nécessité d'entreprendre une quête spirituelle, une quête vers l'Autre. Il faut s'approcher au plus près des images pour remarquer que les édifices sont souvent délabrés, parfois abandonnés, mais demeurent malgré tout. Cette déréliction apparente est contrebalancée par la lumière aveuglante qui inonde les clichés et permet aux églises de retrouver leur fonction première de sanctuaires. Ces oasis pâles, lovées dans des écrins de verdure luxuriante, revêtent une dimension surnaturelle, quasi divine. 
Sally Mann, St. Paul African Methodist Episcopal, 2008-2016,

Gelatin silver print

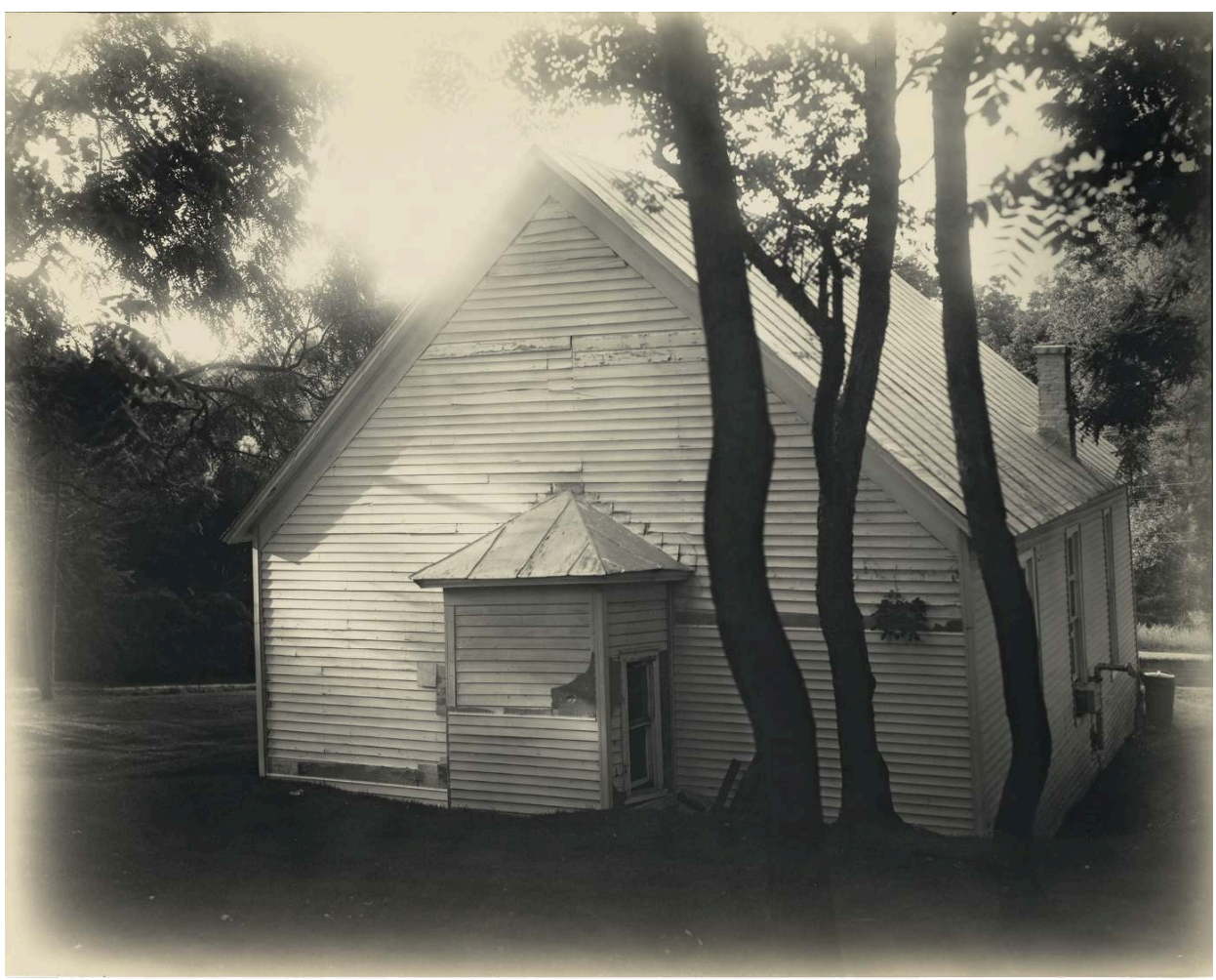

Collection of the artist ( ) Sally Mann

La dernière section, intitulée What Remains (Ce qui reste) en référence au Canto LXXXI d'Ezra Pound (Canto qui appartient à The Pisan Cantos publiés en 1948) aborde à nouveau le thème de la famille, sous un angle radicalement différent. Les paysages édéniques de l'enfance, la maison de vacances, les rivières lumineuses, ont disparu au profit de corps fragmentés. Ces clichés sont autant de memento mori qui enregistrent le passage du temps à même la peau. Les visages flous de ses enfants, devenus de jeunes adultes, remplissent le cadre et le débordent. Les deux triptyques présentés lors de l'exposition évoquent les photographies post-mortem en vogue au XIXe siècle, une époque à laquelle le dernier portrait du défunt figurait souvent aux côtés des vivants dans l'album de famille. Sally Mann ausculte aussi son propre visage et ses variations dans une série d'autoportraits (ambrotypes) réalisés suite à un grave accident de cheval (2006) qui l'empêche de se mouvoir à sa guise. Ce visage, parfois empreint de douleur, devient presque étranger à la photographe d'où la multitude d'expressions, de masques, qu'elle nous livre. Mann se livre à un travail d'introspection et se pose la question de son identité. Enfin, elle explore le corps de Larry, son mari, atteint de dystrophie musculaire, à travers une série de clichés d'un genre très éloigné de la photographie médicale. Elle isole différentes parties de son corps (ses jambes, son buste, son dos) et les transforme en autant de blasons dédiés à l'être aimé. Au-delà des méditations inéluctables que son travail engendre sur la maladie, la vieillesse et la mortalité, ses clichés sont avant tout des actes d'amour, conjurant la peur de l'oubli. Cette obsession du temps qui passe se retrouve également dans les nombreuses références littéraires qui viennent nourrir son travail. Les photographies du corps de Larry sont accompagnées de citations de Vladimir Nabokov (Speak, Memory, 1951), ou 
encore de T. S. Eliot (Four Quartets, publiés entre 1936 et 1942) et de Eudora Welty (The Ponder Heart, 1953). Mais, d'une façon plus large, le travail de Mann est imprégné de littérature, comme le soulignent aussi le titre de l'exposition emprunté à un poème de John Glenday, «Landscape with Flying Man» (Paysage avec homme volant) ou encore son autobiographie, Hold Still: A Memoir with Photographs, publiée en 2015. L'écriture photographique de Mann s'inscrit dans une filiation d'artistes qui ont tous contemplé, disséqué, médité sur le passage inexorable du temps.

«Faulkner de la chambre noire» (ibid. 20), selon Hilton Als, Sally Mann est une conteuse d'histoires. Elle s'empare aussi bien de la petite histoire que de la grande. Mais si son œuvre nous touche autant, c'est parce qu'elle parvient à lui conférer une portée universelle en abordant des thèmes existentiels tels que la vie, la mort, la peur, la haine, l'amour. Quand elle se penche sur la question raciale qui a déchiré le Sud (et le déchire, parfois, encore), elle interroge notre rapport à l'autre. On perçoit chez Mann une responsabilité morale vis-à-vis d'autrui, ce qui rejoint la vision du visage de Levinas pour qui «la relation au visage est d'emblée éthique» (Levinas, 1982, 81). Quand elle photographie les ruines, les églises afro-américaines, les champs de bataille, elle ravive la mémoire et nous exhorte à ne pas oublier. Quand elle fait des portraits de ses proches, elle nous montre que le temps, à l'instar des nombreux cours d'eau qui traversent son œuvre, ne suspend jamais sa course. Elle associe à l'un des clichés de Larry une citation de Nabokov, extraite de son autobiographie Speak, Memory: "Notre existence n'est qu'un bref éclat de lumière entre deux éternités d'obscurité » (Our existence is but a brief crack of light between two eternities of darkness). À travers les fantômes qu'elle ressuscite et à travers les fantômes à venir, Mann nous montre là où réside la mémoire, "Dove sta memoria » pour reprendre les mots de Pound : dans notre capacité à aimer. Sally Mann nous offre une magistrale leçon d'humilité : comme le suggère le titre même de l'exposition, ne sommes-nous pas que de " passage »?

\section{Autour de l'exposition}

« Sally Mann : l'image élégiaque. » Intervenants : Etienne Hatt, Clara Chichin, Géraldine Chouard et Héloïse Conésa. Mis en ligne le 15/07/2019. Page consultée le 18/07/2019. http://lemagazine.jeudepaume.org/2019/07/sally-mann/

\section{BIBLIOGRAPHIE}

NABOKOV, Vladimir. Speak, Memory : An Autobiography Revisited. London : Penguin Books, 1998 (1967).

LEVINAS, Emmanuel. Éthique et infini. Paris : Fayard/France Culture, 1982.

MANN, Sally. Immediate Family. London : Phaidon, 1992. 
Sally Mann : Mille et un passages, catalogue d'exposition. Sous la direction de Sarah GREENOUGH et Sarah KENNEL. Avec des essais de Hilton ALS, Malcolm DANIEL et Drew Gilpin FAUST. Paris : Editions Xavier Barral, 2019.

INDEX

Thèmes : Trans'Arts

\section{AUTEUR}

FAUSTINE RONDIN

Université de Caen 